Draft Version OCtOBER 25, 2018

Preprint typeset using $\mathrm{L}^{\mathrm{A}} \mathrm{T} \mathrm{E}$ X style emulateapj v. 11/10/09

\title{
LATE-TIME DETECTIONS OF THE X-RAY AFTERGLOW OF GRB 060729 WITH CHANDRA - THE LATEST DETECTIONS EVER OF AN X-RAY AFTERGLOW
}

\author{
Dirk Grupe ${ }^{1}$ Grupe@astro.psu.edu, David N. Burrows ${ }^{1}$, Xue-Feng Wu $^{1,2}$, Xiang-Yu Wang ${ }^{3,1}$, Bing Zhang ${ }^{4}$, \\ En-Wei Liang ${ }^{5}$, Gordon Garmire $^{1}$, John A. Nousek ${ }^{1}$, Neil Gehrels ${ }^{6}$ George R. Ricker $^{7}$, Marshall W. Bautz \\ Draft version October 25, 2018
}

\begin{abstract}
We report on 5 Chandra observations of the X-ray afterglow of the Gamma-Ray Burst GRB 060729 performed between 2007 March and 2008 May. In all five observations the afterglow is clearly detected. The last Chandra pointing was performed on 2008-May-04, 642 days after the burst - the latest detection of a GRB X-ray afterglow ever. A reanalysis of the Swift XRT light curve together with the three detections by Chandra in 2007 reveals a break at $\sim 1.0$ Ms after the burst with a slight steepening of the decay slope from $\alpha=1.32$ to 1.61. This break coincides with a significant hardening of the X-ray spectrum, consistent with a cooling break in the wind medium scenario, in which the cooling frequency of the afterglow crosses the X-ray band. The last two Chandra observations in 2007 December and 2008 May provide evidence for another break at about one year after the burst. If interpreted as a jet break, this late-time break implies a jet half opening angle of $\sim 14^{\circ}$ for a wind medium. Alternatively, this final break may have a spectral origin, in which case no jet break has been observed and the half-opening angle of the jet of GRB 060729 must be larger than $\sim 15^{\circ}$ for a wind medium. We compare the X-ray afterglow of GRB 060729 in a wind environment with other bright X-ray afterglows, in particular GRBs 061121 and 080319B, and discuss why the X-ray afterglow of GRB 060729 is such an exceptionally long-lasting event.

Subject headings: gamma rays: burst, X-rays: burst, X-rays: individual (GRB 060729), Swift, Chandra
\end{abstract}

\section{INTRODUCTION}

Gamma-Ray Bursts (GRBs) are the most energetic transient events in the Universe. The most accepted model to explain this phenomenon is the "fireball" model (e.g. Mészáros 2006; Zhang \& Mészáros 2004) and the progenitor of long GRBs are believed to be massive stars on the order of 30 solar masses or more. The isotropic energies inferred from the observed fluences are often of order $10^{53}-10^{54}$ ergs. On the other hand, typical supernova explosions are of the order of only $10^{51}$ ergs. One way to solve this "energy problem" is by assuming that the radiation is collimated into a jet. One prediction of the "fireball" model is that the afterglow decay rate increases when the relativistic beaming angle equals or exceeds the physical jet opening angle as the jet decelerates in the surrounding medium (e.g. Rhoads 1999; Sari et al. 1999). This can be seen as an achromatic jet break in the light curve, with a typical decay slope after the jet break of order $\alpha=2$ (e.g. Zhang et al. 2006; Nousek et al. 2006). The time of this jet break can be used to infer the jet opening angle by e.g. the relation

\footnotetext{
${ }^{1}$ Department of Astronomy and Astrophysics, Pennsylvania State University, 525 Davey Lab, University Park, PA 16802

2 Purple Mountain Observatory, Chinese Academy of Sciences, Nanjing 210008, China

3 Department of Astronomy, Nanjing University, Nanjing 210093, China

${ }^{4}$ Department of Physics, University of Nevada, Las Vegas, NV 89154

${ }^{5}$ Department of Physics, Guangxi University, Nanning 530004, China

${ }^{6}$ Astrophysics Science Division, Astroparticle Physics Laboratory, Code 661, NASA Goddard Space Flight Center, Greenbelt, MD 20771

${ }^{7}$ Massachusetts Institute of Technology, 77 Massachusetts Av., Cambridge, Ma 02139-4307
}

given by Frail et al. (2001). The measurement of the jet break time is therefore most critical for understanding the energetics of GRBs.

Before the launch of the Swift Gamma-Ray Burst Explorer mission (Gehrels et al. 2004), putative jet breaks were found at optical or radio wavelengths, typically a few days after the burst (e.g. Frail et al. 2001). Since its launch, Swift has detected roughly 400 bursts (end of 2008) and typically observes them for up to one or two weeks after the trigger. For the majority of these bursts, jet breaks have not been detected (e.g. Burrows \& Racusin 2007; Willingale et al. 2007; Liang et al. 2008; Racusin et al. 2009). However, one reason could be that jet breaks occur in X-rays at much later times than previously thought and the followup observations by Swift are not late enough in time to detect a jet break, as studies by Willingale et al. (2007) and Sato et al. (2007) suggest. As an alternative, Curran et al. (2008) suggested that the jet breaks are hidden and the light curves are mis-interpreted as a single power law decay although a jet break is there. Only a handful of afterglows have been followed for more than a month by Swift, because usually the X-ray afterglow fades below the Swift X-Ray telescope (XRT; Burrows et al. 2005) detection limit $\sim 10^{-14} \mathrm{ergs} \mathrm{s}^{-1}$ $\mathrm{cm}^{-2}$ roughly a week after the trigger. One of these exceptions is the bright X-ray afterglow of GRB 060729 which was detected by Swift XRT even 125 days after the burst (Grupe et al. 2007).

The Swift Burst Alert Telescope (BAT; Barthelmy 2005) triggered on GRB 060729 on 2006 July 29, 19:12:29 UT (Grupe et al. 2007) and a redshift of $z=0.54$ was measured (Thoene et al. 2006). The XRT and the UV/Optical Telescope (UVOT; Roming et al. 2005), 
started observing the burst about 2 minutes after the trigger. The Swift UVOT was able to follow this afterglow in the UVW1 filter up to 31 days after the BAT trigger. In X-rays Swift's XRT was still detecting the X-ray afterglow of GRB 060729 at the end of 2006 November, 125 days after the burst (Grupe et al. 2007). However, by 2006 December the Swift-XRT detection limit was reached and only a $3 \sigma$ upper limit could be given for the $63.5 \mathrm{ks}$ exposure time obtained in December 2006. By that time the X-ray afterglow of GRB 060729 did not show any clear evidence for a jet break, giving a lower limit on the jet opening angle of $\theta=28^{\circ}$ (Grupe et al. 2007) based on the assumption of a constant circumburst medium. In order to extend the light curve of this exceptional X-ray afterglow, we observed it five times with Chandra ACIS in 2007 and 2008.

We report on the detections of the X-ray afterglow of GRB 060729 (Grupe et al. 2007) with Chandra up to 642 days after the burst - the latest detection ever of an X-ray afterglow of a GRB at cosmological distance. Previously the burst with the latest detection of an X-ray afterglow was GRB 030329 (Tiengo et al. 2003, 2004), which had a detection 258 days after the burst by XMM-Newton. Our paper is organized as follows: in $\$ 2$ the observations and data reduction are explained, in $\oint[3$ the measurements of

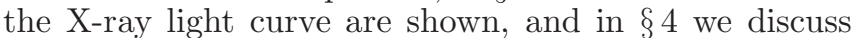
the implications of this light curve. Throughout this paper the X-ray flux dependence on time and frequency is defined as $F \propto t^{-\alpha} \nu^{-\beta}$. Luminosities are calculated assuming a $\Lambda$ CDM cosmology with $\Omega_{\mathrm{M}}=0.30, \Omega_{\Lambda}=0.70$ and a Hubble constant of $H_{0}=71 \mathrm{~km} \mathrm{~s}^{-1} \mathrm{Mpc}^{-1}$ corresponding to a luminosity distance $d_{L}=3064 \mathrm{Mpc}$ for GRB 060729.

\section{OBSERVATIONS AND DATA REDUCTION}

Chandra observed GRB 060729 three times between 2007 March 16 and 2007 June 30. Two very late-time Chandra observations were performed in 2007 December/2008 January for $72.7 \mathrm{ks}$ and in 2008 April/May for $117.3 \mathrm{ks}$. Due to pitch angle constraints some of these had to be split into several visits. All observations, with start and end times and exposure times, are listed in Table1.

All of these observations were performed with the standard $3.2 \mathrm{~s}$ readout time in Very Faint mode on the onaxis position on the back-illuminated ACIS-S3 CCD. Data reduction was performed with the Chandra analysis software CIAO version 4.0 and the calibration database CALDB version 3.4.3. In order to reduce the ACIS particle background all Chandra stage 1 event data were reprocessed using CIAO acis_process_events with the VF mode cleaning. Only ACIS grades $0,2,3,4$, and 6 were selected for further analysis. The background was further reduced by using only photons in the $0.5-8.0 \mathrm{keV}$ energy range. Before further analysis, the observations were combined into one event file each using the CIAO task merge_all. Source photons were selected in a circle with a radius $r=1^{\prime \prime}$ and background photons in a closeby source-free region with a radius $r=10^{\prime \prime}$. Count rates were converted into fluxes by using PIMMS version $3.9 \mathrm{~b}$ using the parameters from the spectral fits to the Swift data after the break at $1 \mathrm{Ms}$ after the burst (see below) with an absorption column density $N_{\mathrm{H}}=1.34 \times 10^{21}$ $\mathrm{cm}^{-2}$ and an X-ray spectral index $\beta_{\mathrm{X}}=0.89$.

A description of the reduction and analysis of the Swift data can be found in Grupe et al. (2007). For display purposes and fitting the late-time light curve we rebinned the Swift XRT Photon Counting data with 250 counts per bin for the times up to $2 \mathrm{Ms}$ after the burst and 100 counts per bin for the times thereafter. Spectral analyses were performed for the times $300-800 \mathrm{ks}$ and $T>$ $1 \mathrm{Ms}$ after the burst. Source photons were collected in a circle with $r=23.5$ and background photons with $r=96^{\prime \prime}$ with grade selection $0-12$. The response matrix swxpc0to12s0_20010101v010.rmf was used. The spectra were rebinned with 20 counts per bin for the $300-800 \mathrm{ks}$ after the burst spectrum and 15 counts per bin for the spectrum with $T>1 \mathrm{Ms}$. The spectra were analyzed with XSPEC version 12.4.0x (Arnaud 1996). To search for changes in the X-ray spectrum we applied a hardness ratio study 8 segment by segment. Because of the low-number statistics in some of the later segments of the Swift XRT and all Chandra observations, we applied Bayesian statistics to determine the hardness ratios as described by Park et al. (2006).

\section{RESULTS}

\subsection{Temporal breaks}

The late-time X-ray light curve including the five Chandra pointings is shown in Figure1. We ignore the first day of the Swift observation because it is not relevant for the study of the late-time light curve. The early light curve and a detailed discussion can be found in Grupe et al. (2007).

The late-time light curve (Figure1) was fitted by several power law and multiply-broken power law models as listed in Table2. Fitting the light curve with the decay slope $\alpha_{3}{ }^{9}$ fixed to 1.32, as reported by Grupe et al. (2007) from the Swift data, gives a very poor result $\left(\chi^{2} / \nu\right.$ $=897 / 46$ ). The fit can be improved by leaving the decay slope as a free parameter (Table2 model 2). This results in a single decay slope of $\alpha_{3}=1.45 \pm 0.01$, but the light curve still deviates significantly at later times from this slope, resulting in an unacceptable $\chi^{2} / \nu=400 / 45$. A broken power law fit to the entire late-time light curve (model 3) reveals a break at about 2 Ms after the burst; in contrast to the result of Grupe et al. (2007), in which we could fit the late-time Swift data with just one decay slope, the addition of the 2007 Chandra data requires a break in the Swift data. The late decay slope, $\alpha_{4}=1.85$, is driven by the last two Chandra observations, while the $\chi^{2}$ is also strongly affected by two very high data points at $\sim 2 \mathrm{Ms}$ and $\sim 5 \mathrm{Ms}$. Making the assumption that these two high points are late-time X-ray flares unrelated to the afterglow of the external shock, we removed them from further fits (see models 4 and 5). We then fit the data between $100 \mathrm{ks}$ and $30 \mathrm{Ms}$ with a broken power law (model 6), obtaining a break time of $T_{\text {break, } 3}=1.01_{-0.22}^{+0.35}$ Ms and slopes of $\alpha_{3}=1.32_{-0.05}^{+0.02}$ and $\alpha_{4}=1.61_{-0.06}^{+0.10}$. This fit is plotted as the dashed line in Figure1.

8 The hardness ratio is defined as $\mathrm{HR}=(\mathrm{H}-\mathrm{S}) /(\mathrm{H}+\mathrm{S})$, where $\mathrm{S}$ and $\mathrm{H}$ are the observed counts in the $0.3-2.0$ and $2.0-10.0 \mathrm{keV}$ energy bands, respectively.

9 We followed the notation as defined in Nousek et al. (2006) and Zhang et al. (2006) where $\alpha_{3}$ is the steep decay slope after the plateau phase. 
The last two Chandra observations deviate from this fit, suggesting a break at about a year after the burst. Because these last two points have very few counts (and consequently large uncertainties), a broken power law fit to the late-time light curve cannot constrain either the break time or the late-time decay slope $\alpha_{5}$ unless at least one parameter is fixed. We therefore approached the question of a final break in steps. A single power law fit to the light curve for $T \geq 1.2 \mathrm{Ms}$ (Table2 model 7 ) gives $\alpha_{4}=1.68 \pm 0.08$ and $\chi^{2} / \nu=12 / 15$. Although this is already an acceptable fit, we investigated the possibility of a late-time break which is expected from GRB theory (c.f. e.g. Zhang et al. 2006; Mészáros 2006). At first we fitted the light curve for $1.2 \mathrm{Ms} \leq T \leq 35 \mathrm{Ms}$ with a single power law (model 8) which results in $\alpha_{4}$ $=1.61_{-0.13}^{+0.07}$ and $\chi^{2} / \nu=6 / 13$. We then fitted a broken power law model to the entire light curve with $T>1.2$ Ms with $\alpha_{4}$ fixed at 1.61 (the best-fit result when the last two Chandra observations are excluded; model 8 in Table 2) to determine whether the data require a very late break in the light curve slope. This fit (Table2 model 9) gives $T_{\text {break }, 4}=41.3_{-5.1}^{+4.2} \mathrm{Ms}$ and $\alpha_{5}=4.65_{-1.34}^{+2.05}$, with $\chi^{2} / \nu=6 / 14$. Figure 3 displays the contour plot between the final break time and the final slope. It shows that they are still not well-constrained. Although the best-fit break time is $41 \mathrm{Ms}$ (2007 November), a break as early as $\sim 26 \mathrm{Ms}$, with a late-time decay slope of $\alpha_{5}=2.5$, is consistent with the data at the $1 \sigma$ level.

In addition to the broken power law fits with a sharp break, the late-time light curve was also fitted by the smoothed double-broken power law model defined by Beuermann et al. (1999). Here we found decay slopes $\alpha_{3}=1.20 \pm 0.07, \alpha_{4}=1.70 \pm 0.05$, and $\alpha_{5}=2.41 \pm 0.26$ with break times at $1.09 \pm 1.01 \mathrm{Ms}$ and $20 \mathrm{Ms}$ (fixed). The smooth parameter is fixed to 3.0 and 2.0 for the breaks at about $1 \mathrm{Ms}$ and $20 \mathrm{Ms}$, respectively. This results in an acceptable fit with $\chi^{2} / \nu=42 / 32$. Possible interpretations of these temporal breaks are discussed in $\$$.

\subsection{Spectral variations}

Temporal breaks are often associated with spectral breaks (e.g. Sari et al. 1998; Mészáros et al. 1998; Zhang et al. 2006). Figure2 displays the Swift XRT count rate and hardness ratio light curves for the interval between $100 \mathrm{ks}$ and $5 \mathrm{Ms}$ after the burst. The hardness ratios are plotted segment by segment. While the hardness ratios before the break at $1 \mathrm{Ms}$ after the burst are of order $\mathrm{HR} \sim 0.3$, after the break the spectrum hardens to $\mathrm{HR} \sim 0.45$ with even harder values at later times.

The spectrum before the $1 \mathrm{Ms}$ break can be fitted with a single absorbed power law with $N_{\mathrm{H}}=\left(1.34_{-0.25}^{+0.27}\right) \times 10^{21}$ $\mathrm{cm}^{-2}$ and an energy spectral slope $\beta_{\mathrm{x}}=1.18 \pm 0.11\left(\chi^{2} / \nu\right.$ $=81 / 82$ ). The spectrum after the $1 \mathrm{Ms}$ break was also fitted by an absorbed single power law model. Leaving the absorption column density as a free parameter, however, results in an increase of the column density, which does not seem plausible. Therefore we fixed the absorption column density to $N_{\mathrm{H}}=1.34 \times 10^{21} \mathrm{~cm}^{-2}$, the value obtained before the break. This fit results in a slightly flatter energy spectral slope $\beta_{\mathrm{x}}=0.89 \pm 0.11$. These values were used in PIMMS to convert the Chandra ACIS-S count rates into the fluxes given in Table 1 and plotted in Figure 1 .
The Chandra data must be analyzed in the Poisson limit, complicating proper analysis of possible spectral variations at very late times. Using the Bayesian approach described by Park et al. (2006), we estimated the hardness ratios in the Chandra datd 10 and their uncertainties, both before and after the break at $38 \mathrm{Ms}$. We obtain mean values of $\mathrm{HR}=-0.39$ for the 2007 MarchJune data (before the final break; 38 counts total) and $\mathrm{HR}=-0.80$ for the very late data (after the final break; 8 counts total), with $85 \%$ confidence limits of $\mathrm{HR}=-0.60$ to -0.17 and $\mathrm{HR}=-1.00$ to -0.58 , respectively. Although this is a suggestion of spectral softening across the final break, we cannot exclude (at the $85 \%$ confidence level) the possibility that the hardness ratio is constant. Note that due to the different energy bands and detector response matrices it is not possible to compare the Swift and Chandra hardness ratios directly.

\subsection{Comparison with other GRBs}

Even though GRB 060729 was one of the brightest bursts detected in X-rays, it is not the brightest one so far seen during the Swift mission. GRB 061121 was about 2-3 times brighter when the Swift-XRT started observing it (Page et al. 2007), but by a day after the burst it was an order of magnitude fainter than GRB 060729 and was not detected after $2 \mathrm{Ms}$ post-burst. The second brightest X-ray afterglow so far seen by Swift was GRB 080319B (Racusin et al., 2008). Figure 4 displays the observed count rate light curves of GRBs 060729, 061121, and 080319B. Even though GRBs 061121 and 080319B appear to be much brighter than GRB 060729 until about $20 \mathrm{ks}$ after the trigger, the long plateau phase in GRB 060729 makes it the brightest X-ray afterglow about half a day after the trigger. GRB 061121 already displays a break from the plateau to the second steep decay phase at $2.2 \mathrm{ks}$ after the burst followed by an even steeper decay at $30 \mathrm{ks}$ with a decay slope of $\alpha=1.5$ (Page et al., 2007). This earlier break compared to GRB 060729, which broke at about $60 \mathrm{ks}$ after the burst, and the steeper decay slope of 1.5 compared to 1.3 in GRB 060729 made GRB 061121 disappear much faster than GRB 060729. The "naked-eye" burst 080319B on the other hand does not even show a noticeable plateau phase and decays rather quickly with late-time decay slopes of $\alpha_{\mathrm{X}}=1.17$ and 2.61 before and after the late-time jet break at $T=9.5 \times 10^{5}$ s.

We can ask how the intrinsic rest-frame $2-10 \mathrm{keV}$ luminosity light curve of GRB 060729 compares to the bursts shown in Figure 1 in Nousek et al. (2006). Figure 5 displays GRBs 060729, 061121, and 080319B with most of the bursts shown in Figure 1 in Nousek et al. (2006) in the rest-frame. The plot shows that during the plateau phase GRB 060729 was not that luminous. As a matter of fact it was a factor of about 10 to 100 less luminous than bursts such as GRBs 061121 or 080319B. Nevertheless after a few days in the rest-frame, GRB 060729 becomes the most luminous X-ray afterglow in the 2 - 10 keV band.

Compared with other GRBs shown in Figure5, the total energy output in the apparent rest-frame $2-10 \mathrm{keV}$

\footnotetext{
10 We defined the Chandra hardness ratio as $\mathrm{HR}=(\mathrm{H}-\mathrm{S}) /(\mathrm{H}+\mathrm{S})$, where $\mathrm{S}$ and $\mathrm{H}$ are the counts in the $0.5-2.0$ and $2.0-8.0 \mathrm{keV}$ bands, respectively.
} 
band of $E_{2-10 \mathrm{keV}}=7 \times 10^{52}$ ergs makes GRB 060729 one of the most energetic X-ray afterglows ever detected. In the 2-10 keV band, only GRBs 061121 and 080319B appear to be more energetic with $E_{2-10 \mathrm{keV}}=1 \times 10^{53}$ ergs and $2 \times 10^{54}$ ergs, respectively. However, if we attempt to correct for the jet opening angle, the picture changes. With jet opening angles of $4^{\circ}$ and $0.4^{\circ}$ as inferred for GRBs 061121 and 080319B, respectively (Page et al. 2007; Racusin et al. 2008), the beamingcorrected energies are $2.4 \times 10^{50}$ and $4.0 \times 10^{50}$ ergs, respectively. For GRB 060729, however, assuming a jet half opening angle of $14^{\circ}$ (see next section) the beaming corrected energy is still $2.1 \times 10^{51}$ ergs in the rest frame 2 - $10 \mathrm{keV}$ band.

\section{DISCUSSION}

While our original Swift XRT light curve after the plateau phase seems to be consistent with a single power law decay (Grupe et al. 2007), the Chandra data make it apparent that there had to be a break at about $1 \mathrm{Ms}$ after the burst. We showed above that this break coincides with a significant change in the X-ray spectral slope from $\beta_{\mathrm{X}}=1.2$ before and 0.9 after that break. Unfortunately, as mentioned in Grupe et al. (2007), Swift could only follow the afterglow in the UVOT with the W1 filter up to a month after the burst due to bright stars in the UVOT field which caused some scatter in the UVOT and an enhanced background at the position of GRB 060729.

According to Model 9 (excluding the two X-ray flare points at $2 \mathrm{Ms}$ and $5 \mathrm{Ms}$ ) in Table 2, the late time $\mathrm{X}$ ray afterglow is described by $F_{\nu_{X}} \propto t^{-\alpha_{i}} \nu_{X}^{-\beta_{i}}$, where (1) $\alpha_{3}=1.32_{-0.05}^{+0.02}, \beta_{3}=1.18 \pm 0.11$ for $105 \mathrm{ks}<t<$ $T_{\text {break }, 3}=1.01_{-0.22}^{+0.35} \mathrm{Ms} ;(2) \alpha_{4}=1.61_{-0.06}^{+0.10}, \beta_{4}=0.89 \pm$ 0.11 for $T_{\text {break }, 3}<t<T_{\text {break }, 4}=4.13_{-0.51}^{+0.42} \times 10^{7} \mathrm{~s} ;(3)$ $\alpha_{5}=4.65_{-1.34}^{+2.05}$ for $t>T_{\text {break }, 4}$.

The closure relation for $0.1 \mathrm{Ms}<t<1.0 \mathrm{Ms}$ results in $\alpha_{3}-1.5 \beta_{3}=-0.46 \pm 0.17$, consistent with both the ISM and wind models with theoretical expectation of $\alpha_{3}-1.5 \beta_{3}=-0.5$ if $\nu_{X}>\max \left\{\nu_{c}, \nu_{m}\right\}$, so $\alpha_{3}=(3 p-2) / 4$ and $\beta_{3}=p / 2$. The power law index of the electron energy distribution is $p=2.43_{-0.07}^{+0.03}$ $(2.36 \pm 0.22)$, derived from $\alpha_{3}\left(\beta_{3}\right)$. The closure relation for $1.0 \mathrm{Ms}<t<41 \mathrm{Ms}$ results in $\alpha_{4}-1.5 \beta_{4}=0.28_{-0.18}^{+0.19}$, moderately consistent with the theoretical expectation of $\alpha_{4}-1.5 \beta_{4}=0.5$ if the environment is a free wind 11 and the spectral regime is $\nu_{m}<\nu_{X}<\nu_{c}$, so $\alpha_{4}=(3 p-1) / 4$ and $\beta_{4}=(p-1) / 2$. In other words, the break at $\sim 1.0 \mathrm{Ms}$ can be interpreted as a cooling break in the wind medium scenario in which the cooling frequency $\nu_{c}$ crosses the $\mathrm{X}$ ray band. The power law index of the electron energy distribution derived from the value of $\alpha_{4}$ (the spectral index of this epoch has a relatively large uncertainty) is $p=2.48_{-0.08}^{+0.13}$, quite consistent with the value derived during the previous epoch. Therefore, a wind model is preferred from the observations before $t=41 \mathrm{Ms}$, breaking the degeneracy of the wind and ISM models which

11 For the ISM model, the closure relation is $\alpha_{4}-1.5 \beta_{4}=-0.5$ or 0.0 , which can be excluded at the $5 \sigma$ and $2 \sigma$ confidence level, respectively. Even if we adopt $\alpha_{4}-1.5 \beta_{4}=0$, the derived $p \sim 3.15_{-0.08}^{+0.13} / 2.78 \pm 0.22$ from the temporal/spectral index is inconsistent with the $p$ value derived with $\alpha_{3}$ and $\beta_{3}$ at the earlier stage. are both consistent with the earlier data Grupe et al. 2007).

The X-ray afterglow light curve for $t>2.75 \times 10^{5} \mathrm{~s}$ excluding the two flares can be also well fitted by a single smoothed broken power law: (1) $\alpha_{3}=1.31 \pm 0.05$, $\beta_{3}=1.18 \pm 0.11$ for $275 \mathrm{ks}<t<T_{\text {break }, 3}=2.43 \pm 0.79$ Ms; (2) $\alpha_{4}=1.96 \pm 0.09, \beta_{4}=0.89 \pm 0.11$ for $T_{\text {break }, 3}<t$ $\left(\chi_{\nu}^{2} /\right.$ dof $=42 / 32$, smoothness parameter $\left.s=3\right)$. The steepening of the decay at $t=2.4 \mathrm{Ms}$, if not due to the cooling frequency passing through the observing band (which results $\alpha_{4}-\alpha_{3}=0.25$, inconsistent with the observation), should originate from the post-jet-break evolution. The change of the temporal decay index, $\alpha_{4}-\alpha_{3}=0.65 \pm 0.10$, is consistent with a non-spreading jet break in a wind model with $\Delta \alpha=0.5$ (if the jet has significant sideways expansion, then the value of $\alpha_{4}$ should be equal to $p \sim 2.4-2.5)$. However, the spectral hardening around the break time can not be well interpreted in such a model. Furthermore, the transition of a jet break in the wind medium usually takes two orders of magnitude in time (Kumar \& Panaitescu 2000), which is inconsistent with the observation of GRB 060729. In conclusion, the temporal break at $t \sim 1-3 \mathrm{Ms}$ is probably not a jet break.

There are two possible interpretations for the last tentative light curve break at $t=41 \mathrm{Ms}$, as follows: a jet break, or a spectral break in a spherical model. We discuss these next, after which we consider the implications of the long plateau phase.

\subsection{Jet model}

The jet + wind model predicts $\alpha_{5}=p \sim 2.4-2.5$ for a sideways expanding jet or $\alpha_{5}=\alpha_{4}+0.5 \sim 2.1$ for a non-sideways expanding jet. The value of the modelpredicted temporal index after the jet break thus can not be excluded at even the $1 \sigma$ confidence level (see Fig. 3). Recently Zhang \& MacFadyen (2009) have performed two dimensional simulations and calculations of GRB afterglow hydrodynamics and emission. They showed that the sideways expansion of GRB jets can be neglected during the relativistic phase (for the sideways expansion of GRB jets see also Kumar \& Granot 2003, Granot \& Kumar 2003) and that the change in decay slope was larger than predicted analytically. Their results may further alleviate the above problem of the relatively shallow theoretical slope compared with the observed steep slope. However, the spectral softening revealed by the hardness ratio evolution around this break time somewhat disfavors the jet break interpretation.

If we interpret this break as a jet break, then a half-opening angle of the jet can be inferred (e.g., Chevalier \& Li 2000). Under the thin-shell approximation for the post-shocked fluid of a relativistic blast wave, the conservation of energy (neglecting the initial baryon loading in the fireball) reads

$$
E_{k, \text { iso }}=M_{\mathrm{sw}} \Gamma^{2} c^{2}=\text { constant }
$$

where $\Gamma$ is the bulk Lorentz factor of the downstream fluid just behind the shock front and $\Gamma_{s}=\sqrt{2} \Gamma$ is the Lorentz factor of the shock. The swept-up circum-burst mass by the blast wave is

$$
M_{\mathrm{sw}}=\int_{0}^{R} 4 \pi r^{2} n(r) m_{p} d r=\frac{4 \pi}{3-k} A R^{3-k} m_{p},
$$


where the environmental density $n(r)=A r^{-k}$, and $A=$ $n_{0}$ for the ISM case $(k=0)$ and $A=3 \times 10^{35} \mathrm{~cm}^{-1} A_{*}$ for the stellar wind case $(k=2)$.

The evolution of the shock radius $R$ measured in the observer's frame is $d R=2 \Gamma_{s}^{2} c d t /(1+z)$, therefore,

$$
R=\left[\frac{(3-k)(4-k) E_{k, \text { iso }} t}{(1+z) \pi A m_{p} c}\right]^{1 /(4-k)},
$$

and

$$
\Gamma=\left[\frac{(3-k) E_{k, \text { iso }}}{4 \pi A R^{3-k} m_{p} c^{2}}\right]^{1 / 2} .
$$

Inserting the inferred values of the physical parameters, we have

$$
\begin{gathered}
R=1.2 \times 10^{19} E_{k, \text { iso }, 54}^{1 / 4} n_{0,-1}^{-1 / 4}\left(\frac{t}{41 \mathrm{Ms}}\right)^{1 / 4} \mathrm{~cm} \\
\Gamma=1.0 E_{k, \text { iso }, 54}^{1 / 8} n_{0,-1}^{-1 / 8}\left(\frac{t}{41 \mathrm{Ms}}\right)^{-3 / 8},
\end{gathered}
$$

for the ISM case, and

$$
\begin{gathered}
R=1.0 \times 10^{20} E_{k, \text { iso }, 54}^{1 / 2} A_{*,-1}^{-1 / 2}\left(\frac{t}{41 \mathrm{Ms}}\right)^{1 / 2} \mathrm{~cm}, \\
\Gamma=4.0 E_{k, \text { iso }, 54}^{1 / 4} A_{*,-1}^{-1 / 4}\left(\frac{t}{41 \mathrm{Ms}}\right)^{-1 / 4}, \\
\theta_{\text {jet }}=14.0^{\circ} E_{k, \text { iso }, 54}^{-1 / 4} A_{*,-1}^{1 / 4}\left(\frac{t}{41 \mathrm{Ms}}\right)^{1 / 4},
\end{gathered}
$$

for the wind case. The values of $E_{k \text {,iso }}$ and $A_{*}$ adopted here can be found below. We adopt the convention of $Q_{x}=Q / 10^{x}$ in cgs units. Unless $n_{0} \ll 0.1 \mathrm{~cm}^{-3}$, the jet has already decelerated to be non-relativistic $(\Gamma \sim 2)$ while the fact that there was no jet break before $t=4.1 \times$ $10^{7} \mathrm{~s}$ argues against the jet in an ISM medium. In other words, the outflow of GRB 060729 is likely spherical if the circum-burst medium is ISM. However, in this way the temporal break at $T_{\text {break,4 }}$ can not be explained with the hydrodynamic/geometry effect.

At such a late time, the jet may also enter the nonrelativistic phase in a stellar wind medium and the hydrodynamics is described by the self-similar Sedov - von Neumann - Taylor solution. The non-relativistic transition time is (Waxman 2004)

$$
t_{\mathrm{SNT}}=2.7 E_{\mathrm{jet}, 52} A_{*}^{-1} \mathrm{yr},
$$

where $E_{\text {jet }} \simeq E_{k, \text { iso }} \theta_{\text {jet }}^{2} / 4$ is the beaming-corrected kinetic energy of the outflow. The non-relativistic transition predicts a flattening in the light curve (Huang \& Cheng 2003; Zhang \& MacFadyen 2009). The theoretical temporal slope is $\alpha_{5}=(7 p-5) / 6 \simeq 2.0-2.1$ for $p \sim 2.4-2.5$, which is not inconsistent with the observations if the large error bars of the observed slope are considered. However, the trend of steepening after the

\footnotetext{
12 Zhang \& MacFadyen (2009) suggested that the beginning time of the SNT self-similar evolution is $\sim 5 t_{\mathrm{NR}}$, where $t_{\mathrm{NR}} \sim$ $200 E_{i, \text { iso }, 54} A_{*}^{-1}$ years is the time when a GRB jet with negligible sideways expansion becomes non-relativistic.
}

break contradicts with the trend of flattening when the jet becomes non-relativistic.

The jet is still inside the free wind bubble $\sim 450$ days after the burst in the observer's frame, indicating that the termination shock radius of the wind bubble is larger than $\sim 32$ pc. The size of a GRB progenitor star wind bubble depends on the density and pressure of external interstellar medium, and the mass loss history of the progenitor (Garcia-Segura et al. 1996; Dai \& Wu 2003; Chevalier et al. 2004). The large size of the wind bubble surrounding the progenitor of GRB 060729 suggests (1) a fast and tenuous wind during the RSG phase prior to the Wolf-Rayet phase, (2) the life time of the WolfRayet stage is relatively long, and (3) the density and pressure of the external interstellar medium should be low. Therefore, from modeling the afterglow, the progenitor of GRB 060729 is not likely to be located in a giant molecular cloud or a star-burst environment. Further deep optical observation of the GRB site and its host galaxy may help to test this prediction.

\subsection{Spherical model}

We assume that the initial shock-accelerated electrons at such a late time have a broken power law distribution,

$$
\frac{d N_{e}}{d \gamma_{e}}=N_{\gamma_{e}} \times \begin{cases}\gamma_{e}^{-p_{1}}, & \gamma_{m}<\gamma_{e}<\gamma_{b}, \\ \gamma_{b}^{p_{2}-p_{1}} \gamma_{e}^{-p_{2}}, & \gamma_{b}<\gamma_{e},\end{cases}
$$

where $p_{1}=p \sim 2.4$ is the low energy power law index and $p_{2}$ is the high energy power law index, $\gamma_{b}$ is the break Lorentz factor of electrons. This assumption is much more realistic than the single power law assumption, especially for the late time when the shock Lorentz factor decreases to the order of unity/the shock is no longer ultra-relativistic (e.g., Hededal et al. 2004; Niemiec \& Ostrowski 2006; Spitkovsky 2008). For simplicity, we assume $R_{b}=\gamma_{b} / \gamma_{m}$ remains a constant in time (Li \& Chevalier 2001). In this scenario, $t_{c}\left(\nu_{c}=\nu_{X}\right)$ is equal to $1.3 \times 10^{6} \mathrm{~s}, t_{b}\left(\nu_{b}=\nu_{X}, \nu_{b}\right.$ is the typical synchrotron frequency of $\gamma_{b}$ electrons) is equal to $4.1 \times 10^{7}$ s. The last steep decay segment can be described with $\alpha_{5}=\left(3 p_{2}-1\right) / 4$ and $\beta_{5}=\left(p_{2}-1\right) / 2$. The high energy power index is therefore derived to be $p_{2}=6.53_{-1.79}^{+2.73}$, and the inferred spectral index is $\beta_{5}=2.77_{-0.90}^{+1.37}$. From $t_{m} \leq 10^{5} \mathrm{~s}$ and $t_{b}=4.1 \times 10^{7} \mathrm{~s}$, we constrain the parameter $R_{b} \geq\left(4.1 \times 10^{7} / 10^{5}\right)^{3 / 4} \sim 91$. The above $p_{1} \sim 2.4$, $p_{2} \sim 6.5$ and $R_{b} \geq 91$ are quite similar to those derived for GRBs 991208 and 000301C in Li \& Chevalier (2001).

The synchrotron emission from a spherical relativistic blast wave can be described by (e.g., Chevalier \& Li 2000; $\mathrm{Wu}$ et al. 2005)

$$
\begin{gathered}
\nu_{m}=3.7 \times 10^{12} \epsilon_{e,-0.5}^{2} \epsilon_{B,-2.5}^{1 / 2} E_{k, \text { iso }, 53}^{1 / 2} t_{5}^{-3 / 2} \mathrm{~Hz} \\
\nu_{c}=3.4 \times 10^{13} \epsilon_{e,-0.5}^{-1} \epsilon_{B,-2.5}^{-1 / 2} E_{k, \text { iso }, 53}^{1 / 2} A_{*}^{-2} t_{5}^{1 / 2} \mathrm{~Hz} \\
F_{\nu, \max }=111 \epsilon_{B,-2.5}^{1 / 2} E_{k, \text { iso }, 53}^{1 / 2} A_{*} t_{5}^{-1 / 2} \mathrm{mJy}
\end{gathered}
$$

where $\epsilon_{e}, \epsilon_{B}$ are the energy equipartition fractions of electrons and magnetic field respectively, $A_{*}$ is the stellar wind parameter. We have considered the cooling effect on $\nu_{c}$ by synchrotron-self-Compton scattering processes. 
The crossing time $t_{m}$ in the optical band $\left(\nu_{\mathrm{opt}} \sim 10^{15}\right.$ $\mathrm{Hz}$ ) by $\nu_{m}$ no later than $t=10^{5} \mathrm{~s}$ gives

$$
\epsilon_{e,-0.5}^{4} \epsilon_{B,-2.5} E_{k, \text { iso }, 53} \leq 7.3 \times 10^{4},
$$

which is easily satisfied, while the crossing time $t_{c}$ in the $\mathrm{X}$-ray band $\left(h \nu_{x} \sim 2 \mathrm{keV}\right)$ by $\nu_{c}$ gives

$$
\epsilon_{e,-0.5} \epsilon_{B,-2.5}^{1 / 2} E_{k, \text { iso }, 53}^{-1 / 2} A_{*}^{2}=2.24 \times 10^{-4} .
$$

The flux density at frequency $\nu_{c}, F_{\nu_{c}}$, should be equal to the observed $2 \mathrm{keV}$ X-ray flux density of $F_{2 \mathrm{keV}} \sim$ $3.5 \times 10^{-5}$ mJy at $t_{c} \sim 10^{6} \mathrm{~s}$, which reads

$$
\epsilon_{e,-0.5}^{-1.7} \epsilon_{B,-2.5}^{-0.7} E_{k, \text { iso }, 53}^{2.4} A_{*}^{-5.2} \sim 8.75 \times 10^{9} .
$$

The above afterglow model parameters can not be tightly constrained due to the lack of more conditions/equations. However, a large late-time isotropic kinetic energy of $E_{k, \text { iso }} \sim 10^{54}-10^{55}$ ergs and a small wind parameter of $A_{*} \sim 0.1$ are favored if we assume $\epsilon_{e} \sim 0.1$ and $\epsilon_{B} \sim 3 \times 10^{-3}$ as inferred from fittings to other afterglows (e.g., Panaitescu \& Kumar 2001; 2002). Within this parameter set, $R_{b}=5.3 \times 10^{4} \epsilon_{e,-0.1}^{-1} \epsilon_{B,-2.5}^{-1 / 4} E_{k, \text { iso }, 54}^{-1 / 4}$ is much higher than those derived from GRBs 991208 and 000301C by Li \& Chevalier (2001).

\subsection{Energy Injection in the shallow decay phase}

A large $E_{k, \text { iso }}\left(\geq 10^{54}\right.$ ergs) at very late time (after the plateau phase ends) can be understood if the early long plateau phase detected both in X-ray and optical bands from a few hundred seconds to $\sim 6 \times 10^{4}$ s since the trigger is interpreted as the period of energy injection, as already discussed in Grupe et al. (2007). Possible scenarios for this enhanced energy injection are refreshed shocks as suggested by Rees \& Mészáros (1998) or the continuous energy injection by the strong magnetic field of a newborn fast-rotating magnetar or black hole as suggested by Dai \& Lu (1998) and Zhang \& Mészáros (2001). In the latter model, the temporal index $q$ of the injected luminosity $\left(L \propto t^{-q}, E_{\text {inj }} \propto t^{1-q}\right)$ can be derived from the observed values of the temporal and spectral indices during the plateau phase (e.g., Table 2 of Zhang et al. 2006). From Table 5 of Grupe et al. (2007), we can obtain $q=2\left(\alpha_{2}-\beta_{2}+1\right) /\left(1+\beta_{2}\right)=-0.037 \pm 0.101$ with $\alpha_{2}=0.14 \pm 0.02$ and $\beta_{2}=1.18 \pm 0.11$ for the X-ray afterglow plateau assuming $\nu_{m}<\nu_{c}<\nu_{X}$. Such a $q$ value is consistent with the theoretical expectation $(q=0)$ of the Poynting-flux dominated injection model. Therefore the isotropic kinetic energy of the shock increases linearly with time, $E_{k \text {,iso }} \propto t$. The prompt isotropic gamma-ray energy release in the $1 \mathrm{keV}-10 \mathrm{MeV}$ band is $E_{\gamma, \text { iso }}=1.6 \times 10^{52}$ ergs (Grupe et al. (2007)). Adopting a typical GRB efficiency of $10 \%-90 \%$, the initial isotropic kinetic energy remaining in the afterglow shock is about the same order of $E_{\gamma \text {,iso }}$, i.e. $E_{k, \text { iso }, \mathrm{i}} \sim 10^{52} \mathrm{ergs}$. After the energy injection is finished, the shock energy $E_{k, \text { iso }}$ is increased by a factor of $\sim 100$ compared to its initial value, so $E_{k \text {,iso }}$ is the order of $\sim 10^{54}$ ergs. The lack of a jet break up to $t=642$ days results a lower limit of the half-opening angle of the GRB jet, i.e.,

$$
\theta_{\mathrm{j}} \geq 15^{\circ} E_{k, \text { iso }, 54}^{-1 / 4} A_{*,-1}^{1 / 4}
$$

which corresponds to a beaming-corrected jet (doublesided) energy of

$$
E_{\text {jet }} \geq 3.4 \times 10^{52} E_{k, \text { iso }, 54}^{1 / 2} A_{*,-1}^{-1 / 2} \text { ergs. }
$$

This makes it one of the most energetic jets ever seen and is the reason why we were able to detect the X-ray afterglow still 642 days after the burst. Note that the energy of a fastest-rotating magnetar is $E_{M}=\frac{1}{2} I \Omega^{2} \leq$ $2 \times 10^{52} I_{45} P_{\mathrm{ms}}^{-2}$ ergs. A massive and fast-rotating black hole as the central engine is thus more likely to provide the required energy.

\section{CONCLUSION}

What makes the X-ray afterglow of GRB 060729 so remarkable is the fact that it was still detected even almost two years after the burst. This exceptional late-time detectability is related to three things: a) with an initial $0.3-10.0 \mathrm{keV}$ flux of almost $10^{-7} \mathrm{ergs} \mathrm{s}^{-1} \mathrm{~cm}^{-2}$ it was one of the brightest afterglows ever detected by Swift, b) its flat decay phase (Nousek et al. 2006; Zhang et al. 2006) extended out to about $60 \mathrm{ks}$ after the burst, and c) the decay slope after that break is about $\alpha=1.3$. Despite breaks at $T \sim 1 \mathrm{Ms}$ and $T \sim 1$ year the afterglow was still detected by Chandra nearly two years after the burst. Bursts like GRBs 060614, 061121, or even 080319B (Mangano et al. 2007; Page et al. 2007; Racusin et al. 2008, respectively) were even brighter in X-rays at about $100 \mathrm{~s}$ after the burst than GRB 060729, but their plateau phases are significantly shorter than that of GRB 060729. They therefore faded more rapidly than GRB 060729 at late times.

Analysis and modeling of the X-ray afterglow of GRB 060729 show that this burst happened in a tenuous wind. This is consistent with the collapsar picture of long GRBs. During the early plateau phase, the energy in the external shock increased by two orders of magnitude. A reanalysis of the Swift XRT light curve together with the three detections by Chandra in 2007 reveal a temporal break at $\sim 1.3 \mathrm{Ms}$ after the burst. The decay slope steepened from $\alpha=1.32$ to $\alpha=1.61$ around this break and the X-ray spectrum hardened in the meanwhile, indicating this break is a cooling break (the cooling frequency of synchrotron radiation crosses the X-ray band). There is another light curve break at $\sim 1.3$ year after the burst tentatively indicated by the last two Chandra detections. This break coincides with a possible spectral softening, suggesting that the break may be of spectral origin, though a hydrodynamic origin (jet break) is also possible. If due to a jet break, then the implied halfopening angle is $\theta_{j} \sim 14^{\circ}$. If due to a spectral break, such a spectral softening could be the result of a very steep power-law distribution of shock-accelerated electrons responsible for the synchrotron radiation. In this case, with no evidence for a jet break up to 642 days after the burst by Chandra the jet half-opening angle must be $\theta_{j}>15^{\circ}$ and the jet energy $E_{j}>3 \times 10^{52} \mathrm{erg}$. Such a large jet energy implies that the central engine must be a fast-rotating massive black hole, not a magnetar.

Our Chandra observations presented here have shown again how important Chandra is for late-time observations of GRB X-ray afterglows. Chandra has already been essential for the detection and non-detection of jet breaks in the X-ray afterglows of the short- 
duration GRBs 050724 and 051221A (Grupe et al. 2006; Burrows et al. 2006, respectively).

We want to thank Sandy Patel and Chryssa Kouveliotou for providing the data for the luminosities of Swift and pre-Swift bursts, Hala Eid for fitting the late-time light curve with the Beuermann et al. model, and Eric Feigelson for useful discussion about statistics. We thank the referee for his/her suggestions/comments which have helped to improve our paper significantly. We are extremely thankful to the whole Chandra team for successfully planning and performing the observations of
GRB 060729. XFW thanks Peter Mészáros, Kenji Toma, Derek Fox, Antonino Cucchiara, and Yizhong Fan for their helpful discussion. This work was also partially supported by NASA NNX 08AL40G (XFW), National Natural Science Foundation of China (grants 10221001, 10403002, 10503012, 10621303, 10633040, and 10873002), and National Basic Research Program of China (973 Program 2009CB824800) (XYW, XFW, and EWL). Swift is supported at Penn State by NASA contract NAS5-00136. This research has been supported by SAO grants SV4-74018, A12 (D.G. and G.G.) and G089056 X (D.G.)

\section{REFERENCES}

Arnaud, K. A., 1996, ASP Conf. Ser. 101: Astronomical Data Analysis Software and Systems V, 101, 17

Barthelmy, S. D., 2005, Space Science Reviews, 120, 143

Beuermann, K., et al., 1999, A\&A, 352, L26

Burrows, D. N., et al., 2005, Space Science Reviews, 120, 165

Burrows, D. N., et al., 2006, ApJ, 653, 468

Burrows, D. N., \& Racusin, J., 2007, Il Nuovo Cimento B, Vol 121, 1273

Chevalier, R. A., \& Li, Z.-Y., 2000, ApJ, 539, 195

Chevalier, R. A., Li, Z.-Y., \& Fransson, C., 2004, ApJ, 606, 369

Curran, P. A., van der Horst, A., \& Wijers, R. A. M. J., 2008, MNRAS, 386, 859

Dai, Z. G., \& Lu, T., 1998, A\&A, 333, L87

Dai, Z. G., \& Wu, X. F., 2003, ApJ, 591, L21

Evans, P. A., et al., 2009, MNRAS, 397, 1177

Frail, D. A., et al., 2001, ApJ, 562, L55

Garcia-Segura, G., Langer, N., \& Mac Low, M-M, 1996, A\&A, 316,133

Gehrels, N., et al., 2004, ApJ, 611, 1005

Granot, J., \& Kumar, P., 2003, ApJ, 591, 1086

Grupe, D., Burrows, D. N., Patel, S. K., Kouveliotou, C., Zhang, B., Mészáros , P., Wijers, R. A. M., \& Gehrels, N., 2006, ApJ, 653, 462

Grupe, D., et al., 2007, ApJ, 662, 443

Hededal, C. B., Haugbølle, T., Frederiksen, J. T., \& Nordlund, A, 2004, ApJ, 617, L107

Huang, Y. F., \& Cheng, K. S., 2003, MNRAS, 341, 263

Kumar, P., \& Panaitescu, A., 2000, ApJ, 541, L9

Kumar, P., \& Granot, J., 2003, ApJ, 591, 1075

Li, Z.-Y., \& Chevalier, R. A., 2001, ApJ, 551, 940

Liang, E.-W., Racusin, J. L., Zhang, B., Zhang, B.-B., \& Burrows, D. N., 2008, ApJ, 675, 528

Mangano, V., et al., 2007, A\&A, 470, 105

Mészáros , P., 2006, Rep. Prog. Phys., 69, 2259

Mészáros , P., Rees, M. J., \& Wijers, R. A. M. J., 1998, ApJ, 499, 301
Niemiec, J., \& Ostroswski, M., 2006, ApJ, 641, 984

Nousek, J., Kouveliotou, C., Grupe, D., Page, K. L., et al., 2006, ApJ, 642, 389

Page, K. L., et al., 2007, ApJ, 663, 1125

Panaitescu, A., \& Kumar, P., 2001, ApJ, 560, L49

Panaitescu, A., \& Kumar, P., 2002, ApJ, 571, 779

Park, T., Kashyap, V. L., Siemiginowska, A., van Dyk, D. A.,

Zezas, A., Heinke, C., \& Wargelin, B. J., 2006, ApJ, 652, 610

Racusin, J. L., et al., 2008, Nature, 455, 183

Racusin, J. L., et al., 2009, ApJ, 698, 43

Rees, M. J., \& Mészáros , P., 1998, ApJ, 496, L1

Rhoads, J. E., 1999, ApJ, 525, 737

Roming, P. W. A., et al., 2005, Space Science Reviews, 120, 95

Sari, R., Piran, T., \& Narayan, R., 1998, ApJ, 497, L17

Sari, R., Piran, T., \& Halpern, J. P., 1999, ApJ, 524, L43

Sato, G., et al., 2007, ApJ, 657, 359

Spitkovsky, A., 2008, ApJ, 682, L5

Thoene, C. C., et al. 2006, GCN Circ. 5373,

http://gcn.gsfc.nasa.gov/gcn3/5373.gcn3

Tiengo, A., Mereghetti, S., Ghisellini, G., Rossi, E., Ghirlanda, G., \& Schartel, N., 2003, A\&A, 409, 983

Tiengo, A., Mereghetti, S., Ghisellini, G., Tavecchio, F., \&

Ghirlanda, G., 2004, A\&A, 423, 861

Troja, E., et al., 2007, ApJ, 665, 599

Waxman, E., 2004, ApJ, 602, 886

Willingale, R., et al., 2007, ApJ, 662, 1093

Wu, X. F., Dai, Z. G., Huang, Y. F., \& Lu, T., 2005, ApJ, 619, 968

Zhang, B., \& Mészáros , P, 2001, ApJ, 552, L35

Zhang, B., \& Mészáros , P., 2004, Int. Jour. Mod. Phys. A, 19, 2385

Zhang, B., et al., 2006, ApJ, 642, 354

Zhang, W. Q., \& MacFadyen, A., 2009, ApJ, 698, 1261 


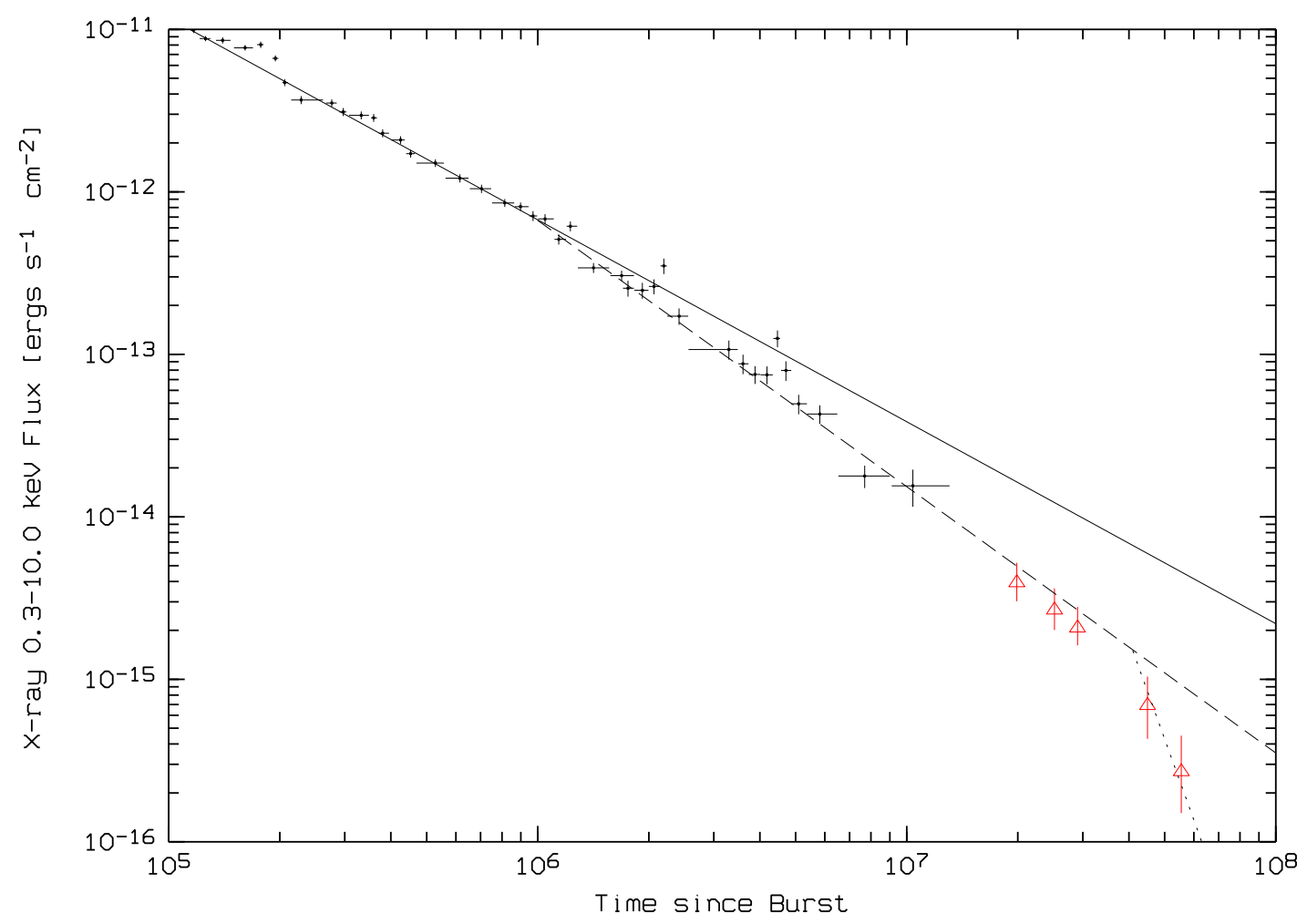

FIG. 1. - Swift-XRT and Chandra ACIS-S light curve of the X-ray afterglow of GRB 060729. The black crosses display the Swift XRT data and the red triangles the Chandra data. The solid line displays the initial decay slope $\alpha_{3}=1.32$ as reported by Grupe et al. (2007), the dashed line the decay slope post-break at $1.0 \mathrm{Ms}$ after the burst with $\alpha_{4}=1.61$ (from model 6 in Table 2), and the dotted line the steep decay after the jet break at $41 \mathrm{Ms}$ after the burst with $\alpha_{5}=4.65$. 


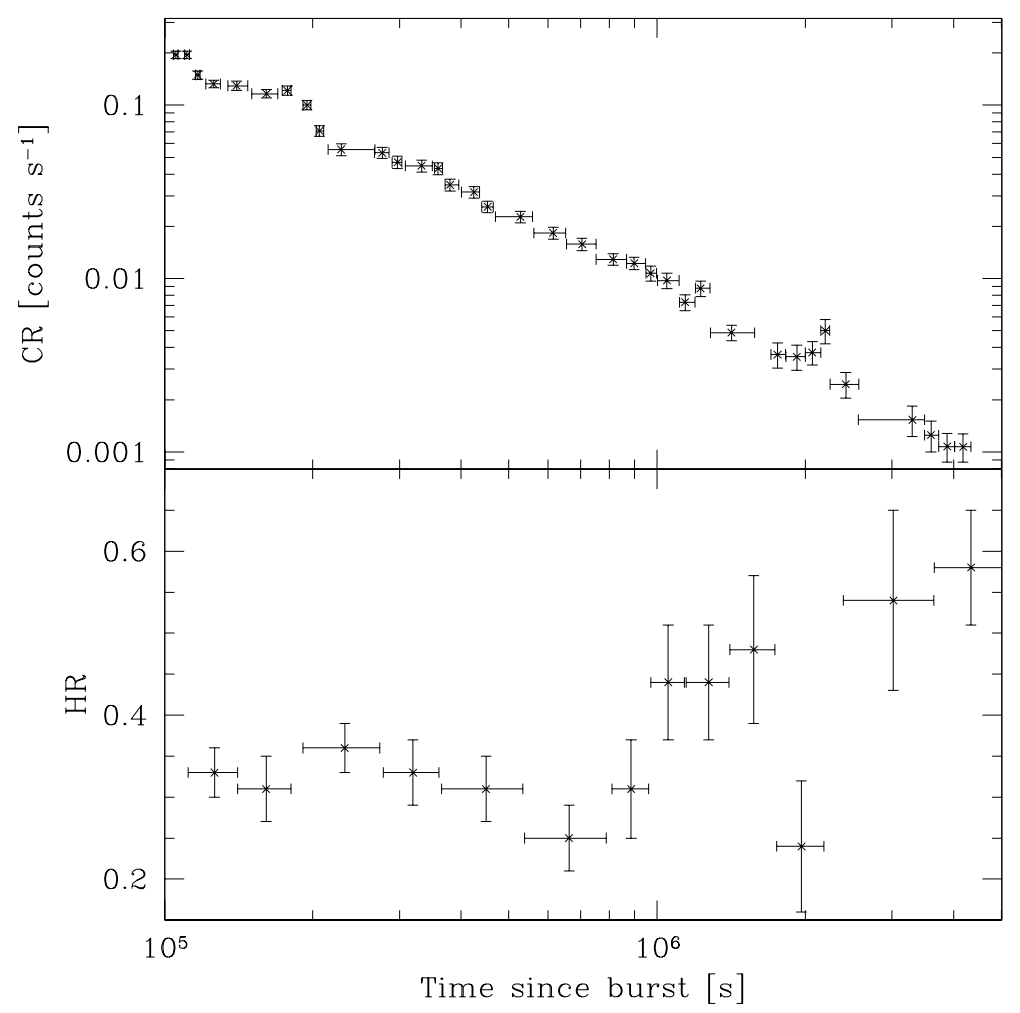

FIG. 2.- Swift-XRT count rate and hardness ratio (as defined in section 3.1, footnote 8) light curves for the time around the break at $1.0 \mathrm{Ms}$ after the burst.

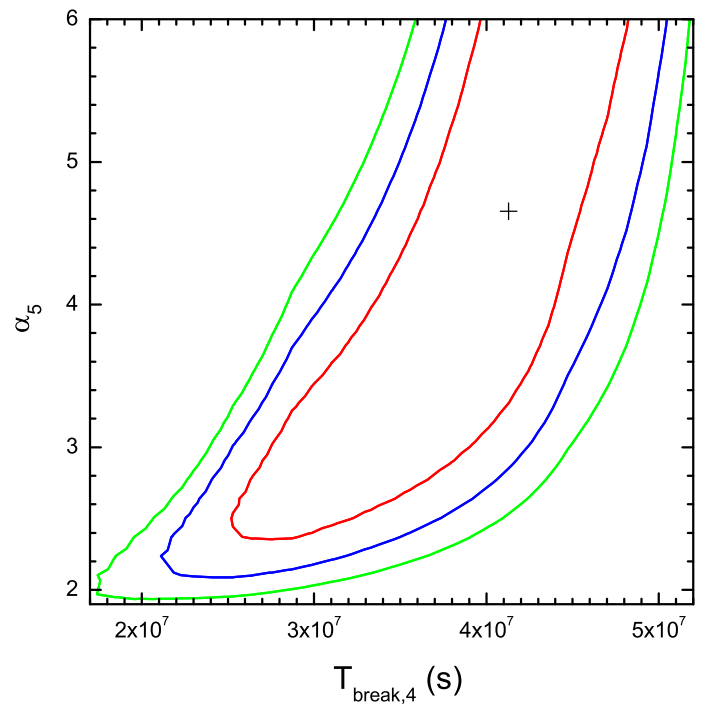

FIG. 3.- Contour plot between the late-time break and decay slope $\alpha_{5}$ of the fit to the late time light curve of GRB 060729 with $T>1.2$ Ms after the burst, excluding the flares at 2 and $5 \mathrm{Ms}$ (Model \#9 in Table2). The lines mark the 1, 2, and $3 \sigma$ confidence levels. 


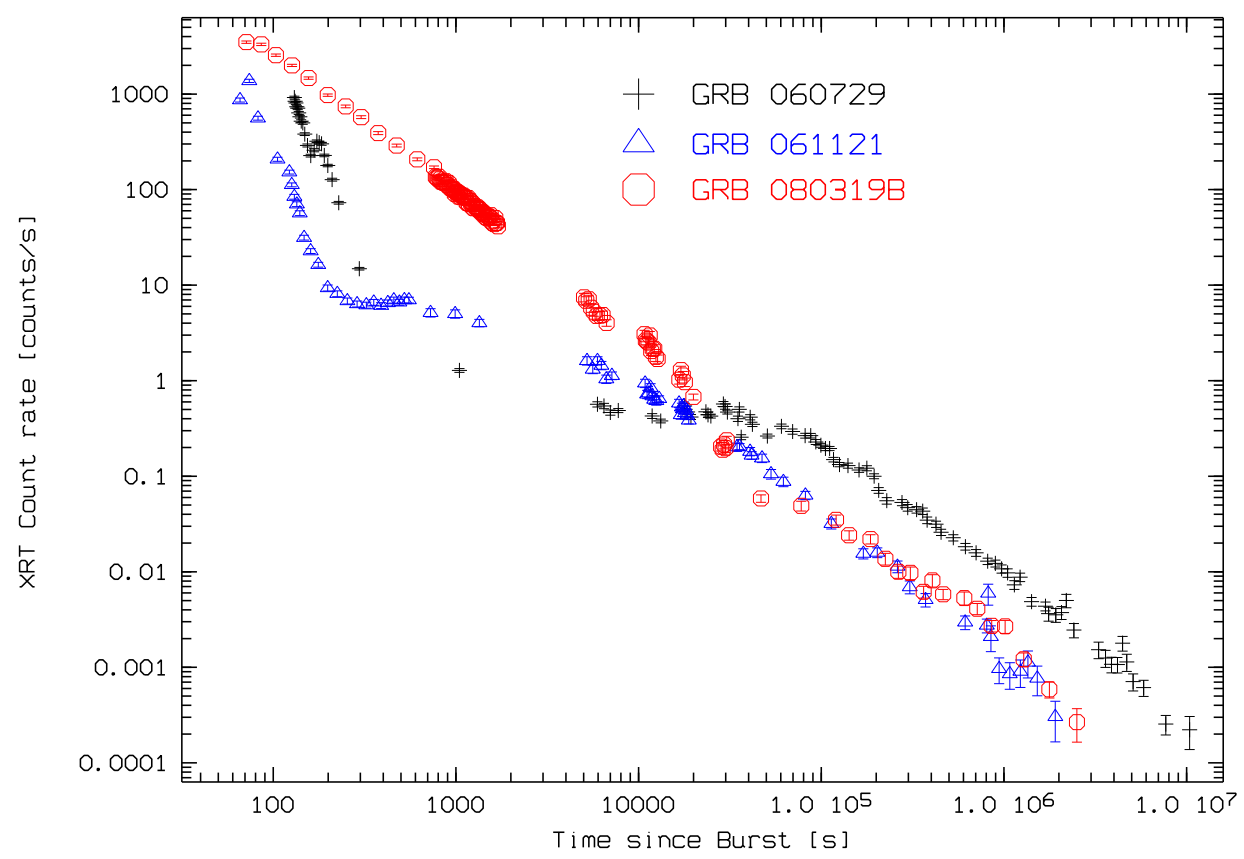

Fig. 4.- Comparison of the observed X-ray light curves of GRBs 060729, 061121, and GRB 080319B 


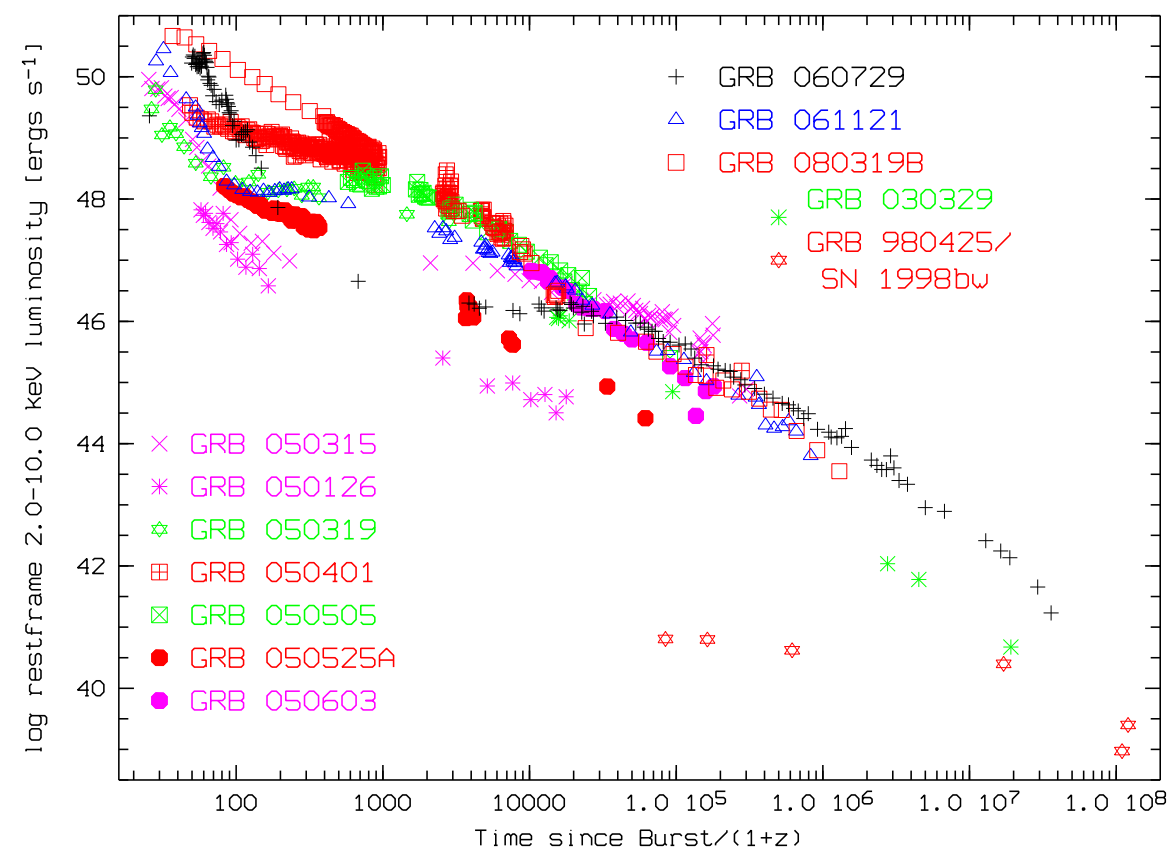

FIG. 5.- Rest frame isotropic equivalent luminosity light curves of several Swift and pre-Swift bursts taken from Nousek et al. (2006) including GRBs 060729, 061121, and 080319B. 
TABLE 1

Chandra ObServation LOG OF GRB 060729

\begin{tabular}{|c|c|c|c|c|c|}
\hline ObsID & T-start ${ }^{1}$ & T-stop ${ }^{1}$ & $\mathrm{~T}_{\exp }^{2}$ & $\mathrm{CR}^{3}$ & $F_{0.3-10.0 \mathrm{keV}^{4}}$ \\
\hline $\begin{array}{l}7567 \\
8541\end{array}$ & $\begin{array}{ll}2007-03-16 & 11: 39 \\
2007-03-17 & 13: 02\end{array}$ & $\begin{array}{ll}2007-03-16 & 19: 57 \\
2007-03-17 & 14: 54\end{array}$ & $\begin{array}{r}27690 \\
4701 \\
\end{array}$ & $3.93_{-0.91}^{+1.28}$ & $4.26_{-0.99}^{+1.39}$ \\
\hline 7568 & 2007-05-16 09:30 & 2007-05-16 20:56 & 40268 & $2.67_{-0.66}^{+0.96}$ & $2.90_{-0.72}^{+1.04}$ \\
\hline 7569 & 2007-06-30 06:19 & 2007-06-30 23:48 & 60400 & $2.07_{-0.45}^{+0.72}$ & $2.25_{-0.49}^{+0.78}$ \\
\hline$\overline{9086}$ & $2007-12-2601: 02$ & $2007-12-2609: 04$ & 27395 & \multirow{4}{*}{$0.69_{-0.26}^{+0.35}$} & \multirow{4}{*}{$\begin{array}{r}0.75_{-0.28}^{+0.38} \\
\text { - }\end{array}$} \\
\hline 9801 & $2007-12-28 \quad 19: 23$ & 2007-12-28 23:59 & 15068 & & \\
\hline 9802 & $2007-12-2918: 57$ & 2007-12-30 01:13 & 20239 & & \\
\hline 9803 & 2008-01-05 01:04 & 2008-01-05 04:23 & 9986 & & \\
\hline 9087 & $2008-04-3023: 09$ & 2008-05-01 08:34 & 32330 & \multirow{4}{*}{$0.26_{-0.12}^{+0.18}$} & \multirow{4}{*}{$0.28_{-0.13}^{+0.19}$} \\
\hline 9811 & 2008-05-01 22:45 & 2008-05-02 07:50 & 30775 & & \\
\hline 9812 & 2008-05-03 15:51 & 2008-05-03 23:35 & 26382 & & \\
\hline 9813 & 2008-05-04 15:07 & 2008-05-04 23:22 & 27810 & & \\
\hline
\end{tabular}

${ }^{1}$ Start and End times are given in UT

2 Observing time given in $\mathrm{s}$

3 Count rate in units of $10^{-4}$ ACIS-S counts $\mathrm{s}^{-1}$ in the $0.5-8.0 \mathrm{keV}$ band

${ }^{4}$ Unabsorbed $0.3-10.0 \mathrm{keV}$ flux in units of $10^{-15} \mathrm{ergs} \mathrm{s}^{-1} \mathrm{~cm}^{-2}$. The count rates were converted assuming $N_{H}=1.34 \times 10^{21} \mathrm{~cm}^{-2}$ and $\beta_{\mathrm{x}}=0.89$.

TABLE 2

Fits to the LATE-Time $\left(\mathrm{T}>10^{5} \mathrm{~s}\right)$ Swift XRT AND Chandra LIGHT CURVE of GRB 060729

\begin{tabular}{|c|c|c|c|c|c|c|}
\hline Model & $\alpha_{3}$ & $T_{\text {break }, 3}{ }^{1}$ & $\alpha_{4}$ & $T_{\text {break }, 4}{ }^{1}$ & $\alpha_{5}$ & $\chi^{2} / \nu$ \\
\hline 1) powl fixed ${ }^{2}$ & 1.32 (fixed) & - & - & - & - & $897 / 46$ \\
\hline 2) powl free ${ }^{3}$ & $1.45 \pm 0.01$ & - & - & - & - & $400 / 45$ \\
\hline 3) bknpowl & $1.32_{-0.01}^{+0.03}$ & $2.08_{-0.20}^{+0.42}$ & $1.85_{-0.06}^{+0.10}$ & - & - & $168 / 43$ \\
\hline 4) powl $^{4}$ & $1.46 \pm 0.04$ & - & - & - & - & $160 / 41$ \\
\hline 5) bknpowl ${ }^{4}$ & $1.32_{-0.05}^{+0.02}$ & $1.23_{-0.40}^{+0.25}$ & $1.70_{-0.07}^{+0.06}$ & - & - & $65 / 39$ \\
\hline 6) bknpowl $T<30 \mathrm{Ms}^{4}$ & $1.32_{-0.05}^{+0.02}$ & $1.01_{-0.22}^{+0.35}$ & $1.61_{-0.06}^{+0.10}$ & - & - & $60 / 37$ \\
\hline 7) powl $T \geq 1.2 \mathrm{Ms}^{4}$ & - & -0.22 & $1.68 \pm 0.08$ & - & - & $12 / 15$ \\
\hline 8) powl $1.2 \mathrm{Ms}<T<35 \mathrm{Ms}^{4}$ & - & - & $1.61_{-0.13}^{+0.07}$ & - & - & $6 / 13$ \\
\hline 9) bknpowl $T \geq 1.2 \mathrm{Ms}^{4,5}$ & - & - & 1.61 (fixed) & $41.3_{-5.1}^{+4.2}$ & $4.65_{-1.34}^{+2.05}$ & $6 / 14$ \\
\hline
\end{tabular}

${ }^{1}$ Break time $T_{\text {break }}$ are given in units of Ms

2 Decay slope fixed to the value given in Grupe et al. (2007)

3 Decay slope parameter left free to vary

${ }_{5}^{4}$ Excluding the two flares at $2 \mathrm{Ms}$ and $5 \mathrm{Ms}$

${ }^{5}$ Error bars of $\alpha_{5}$ and $T_{\text {break,2 }}$ are determined by keeping $\alpha_{4}$ fixed at the best-fit value for model 4 and assuming that $\chi^{2}-\chi_{\text {min }}^{2}$ follows the Gaussian distribution (see Fig. 3). 